
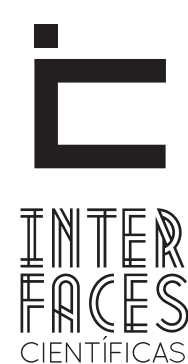

SA ÚDE E AMBIENTE

\title{
O PAPEL DO ENFERMEIRO NO PRÉ-NATAL REALIZADO NO PROGRAMA DE SAÚDE DA FAMÍLIA - PSF
}

Brígida Cabral Souza ${ }^{1}$

Amanda Rafaela Cruz Bernardo ${ }^{2}$

\section{RESUMO}

Este trabalho tem como escopo a análise do papel do enfermeiro na assistência pré-natal realizada na esfera do Programa de Saúde da Família - PSF. Na consecução do objetivo principal, descreve-se a evolução histórica do PSF e das politicas de atenção à saúde da mulher, bem como da relação do profissional da enfermagem com o PSF, especificamente com a realização do pré-natal. 0 exame do tema tem por base a pesquisa bibliográfica e documental que foi realizada mediante artigos, tendo como métodos de abordagem inicial e final o dedutivo e o qualitativo, respectiva-
Licia Santos Santana ${ }^{3}$

mente. Outrossim, o estudo realizado, concentrado na implementação do Programada de Saúde da Família, no desenvolvimento das politicas de atenção à saúde da mulher e, principalmente, na realização do pré-natal pelos profissionais da enfermagem, demonstra a evolução deste profissional, numa perspectiva que the garantia maior autonomia.

\section{PALAVRAS-CHAVE}

Programa de Saúde da Família. Enfermagem. Pré-Natal. 


\section{ABSTRACT}

This work aims to analyze the role of the role of the nurse in prenatal care done in the area of Family Health Program - FHP. To achieve the main objective, describes the historical evolution of the FHP and policy attention to the health of women, and the relationship of the nurse with the FHP, particularly with the completion of prenatal. The examination of the theme is based on bibliographic and documents which was performed by articles, and methods of aprespectively. In addition, the study focused on the implementation of the Family Health Program in the development of policies for women's health and especially the completion of prenatal by nursing professionals, demonstrates the evolution of this view that will guarantee greater autonomy.

\section{KEYWORDS}

Family Health Program. Nurse. Prenatal Care. proach as initial and final deductive and qualitative,

\section{RESUMEN}

Este trabajo tiene como objetivo analizar el papel de la enfermera en la atención prenatal realizado en el ámbito del Programa de Salud de la Familia - PSF. Para lograr el objetivo principal, se describe la evolución histórica del PSF y la atención política a la salud de las mujeres, y la relación del profesional de enfermería con el PSF, en concreto con la realización del prenatal. El examen del tema se basa en la literatura y los documentos que se celebró en los artículos del 2001 al 2011, con los métodos de aproximación inicial y final deductivo y cualitativo, respectivamente. Además, el estudio se centró en la aplicación del Programa de Salud Familiar en el desarrollo de la atención política a la salud de las mujeres y sobre todo de la finalización de los profesionales de enfermería prenatal, muestra la evolución de este punto de vista profesional que garantizará una mayor autonomía.

\section{PALABRAS CLAVE}

Programa de Salud de la Familia. Enfermería. Prenatal.

\section{INTRODUÇ̃̃̃O}

A realização do pré-natal de qualidade influi de forma patente no resultado perinatal e na redução das taxas de mortalidade materna, isto é, certifica a ocorrência de um parto sem intercorrências, inibindo eventuais prejuízos à saúde da mãe e do recém-nascido. (BORGES, 2005).

Nesse contexto, o enfermeiro, membro da equipe multiprofissional de saúde, com o advento do Programa de Saúde da Família - PSF, ganhou um amplo espaço de atuação na assistência pré-natal, desenvolvendo o seu trabalho na unidade de saúde da família e na própria comunidade, contando com o apoio dos agentes comunitários de saúde e auxiliares de enfermagem, profissionais submetidos a sua supervisão.
Esse quadro, no entanto, é fruto de novas perspectivas voltadas a saúde, decorrentes da evolução das políticas públicas, muitas delas impulsionadas pelas recorrentes reivindicações sociais por melhorias nos serviços de saúde.

Nessa linha de entendimento, a importância do enfermeiro ganhou novos contornos. Hodiernamente, este profissional passa a integrar, cada vez mais, em conjunto com outros profissionais, o corpo decisório das equipes de saúde, ganhando credibilidade no corpo social.

Bem por isso, justifica-se o discorrer dos aspectos concernentes a nova ótica sobre a assistência pré-natal no PSF, clarificando as atribuições do profissional 
da enfermagem, pressuposto necessário à qualificação do próprio profissional que atua no âmbito da atenção básica, como dos usuários ali atendidos.

Logo, diante dessa nova temática, o problema posto é saber qual o papel do enfermeiro no cuidado com as gestantes, notadamente na realização do pré-natal no âmbito do PSF.

Não por outro motivo que o objetivo geral é analisar as atribuições do profissional da enfermagem na assistência pré-natal realizada na esfera do Programa de Saúde da Família - PSF. Conseguintemente, são objetivos específicos: descrever o cenário brasileiro, no que pertine as políticas de saúde, à época da implantação do PSF; caracterizar o PSF; definir o profissional da enfermagem; discriminar as atribuições gerais do profissional da enfermagem; estabelecer a relação do enfermeiro com o PSF; especificar a efetivação das políticas de atenção à saúde da mulher; e, por fim, enunciar e explicar as atribuições do profissional da enfermagem na realização do pré-natal no PSF.

\section{METODOLOGIA}

0 presente trabalho tem como base um estudo de revisão, realizada por meio de registros já disponíveis, fruto de pesquisas anteriores, como artigos e manuais técnicos de saúde, utilizando-se destes dados já trabalhados por outros pesquisadores (SEVERINO, 2007, p. 122). A pesquisa documental também é contemplada, mais precisamente no tocante à análise das diversas legislações e regulamentações que fazem referência ao tema em pauta.

Trata-se de um estudo de revisão bibliográfica, abrangente por meio eletrônico e impresso, utilizando os descritores do assunto: programa saúde da fa-
O hodierno papel do enfermeiro na realização do pré-natal no âmbito no PSF, embora aparente ser tema recorrente, ainda algo pouco assimilado pelos profissionais que compõem a equipe multiprofissional de saúde, demandando maior aprofundamento, pois se trata de uma importante ferramenta, diretamente relacionada com a sadia qualidade de vida das mulheres.

Em razão do que restou dito, releva-se a imprescindibilidade da análise das atribuições do profissional da enfermagem no pré-natal realizado no PSF, mormente de suas bases, estruturação, eficiência, aplicação e desenvolvimento ao longo da gravidez. Além disso, o tema proposto tem a sua importância justificada pela relevância teórica e social em torno dos cuidados de saúde.

0 presente estudo, cujo tema situa-se no âmbito da atenção a saúde da mulher, tem o objetivo de ver o papel do enfermeiro no pré-natal realizado no Programa de Saúde da Família - PSF.

mília, enfermagem e pré-natal. Foi obtido a partir de pesquisas realizadas no período de outubro de 2012 a abril 2013. Nesse estudo foi utilizadas bibliografias de acervo próprio e nas bases de dados (Biblioteca Virtual em Saúde - Bireme, Scielo, LILACS).

Finalmente, observa-se que o presente trabalho está disposto, na sequência, com o discorrer acerca do PSF, das atribuições gerais do profissional da enfermagem e das suas atribuições especificas na realização do pré-natal no dito programa. Assim, permite-se a compreensão efetiva do profissional em tela na realização da importante atividade no PSF. 


\section{RESULTADOE DISCUSSÃO}

\section{1 - O PROGRAMA DE SAÚDE DA FAMÍLIA - PSF E AS ATRIBUI- Ç̃̃ES GERAIS DO ENFERMEIRO}

Antes de adentrar na análise meritória do tema deste trabalho, faz-se mister discorrer a respeito do PSF e das atribuições gerais do profissional da enfermagem, possibilitando, assim, o entendimento do conteúdo explanado, em toda sua extensão.

A assistência pública à saúde no Brasil tem como grande marco a Constituição Federal de 1988, notadamente em função da criação do Sistema Único de Saúde - SUS, cuja implantação teve inicio nos anos 90, após a criação da Lei Orgânica da Saúde e da regulamentação por via das várias normas e portarias emitidas pelo Ministério da Saúde - MS.

A implantação do SUS, no entanto, aquela época, foi acompanhada por diversos problemas em sua operacionalização, além de estar envolta em um período de grandes transformações demográficas e epidemiológicas. Esse contexto, decorrente dos diversos problemas, criou um cenário denominado, nos anos 90 , de crise da saúde, demandando um conjunto de modificações que viriam a conceber, momentos depois, o Programa de Saúde da Família - PSF (VIANA e DAL POZ, 1998).

Nessa esteira, como antecessor do PSF, destaca-se a formulação, pelo Ministério da Saúde, do Programa de Agentes Comunitários de Saúde - PACS, que desempenhou um importante papel na conturbada implantação do SUS e, principalmente, na reorganização dos sistemas locais de saúde, conforme ressaltam Viana e Dal Poz (Op. cit.), observe-se:

[...] duas questões tomaram relevância no processo de implantação do PACS: a escolha do agente (envolvendo questões como processo seletivo, capacitação, avaliação, etc.) e as condições institucionais da gestão da saúde no nível local (grau de participação dos usuários; formação dos conselhos de saúde; grau de autonomia da gestão financeira; recursos humanos disponíveis; capacidade instalada disponível, etc.).

Por isso, pode-se afirmar que, pelo fato de o Programa de Agentes Comunitários ter tomado essa forma, ele não se constituiu, então, em apenas mais um programa vertical do Ministério da Saúde (uma ação paralela ao sistema de saúde). Foi também um braço auxiliar na implantação do SUS e na organização dos sistemas locais de saúde. Porque no momento em que a adesão ao município ao PACS passou a exigir certos requisitos - como o funcionamento dos conselhos municipais de saúde, a existência de uma unidade básica de referencia do programa, a disponibilidade de um profissional de nível superior na supervisão e auxílio às ações de saúde e a existência de fundo municipal para receber os recursos do programa - este se tornou, sobretudo, um instrumento de (re)organização dos modelos locais de saúde.

Com o êxito do programa em comento, discutiu-se uma nova proposta, em meados do ano de 1994, visando à inserção de outros profissionais, acabando com o "isolamento" dos agentes comunitários. Destarte, criou-se o Programa de Saúde da Família - PSF, que ganhou outros contornos com o passar do tempo.

O Programa de Saúde da Família, em síntese, é um serviço de primeiro contato, realizado por via dos profissionais da atenção básica ou primária, incumbidos de filtrar os diversos casos e proceder com o encaminhando dos usuários, quando pertinente, aos profissionais da atenção especializada. Em outras palavras, o PSF é a porta de entrada no sistema de saúde, devendo abarcar, entre outras coisas, condições de acessibilidade à população, eliminando-se eventuais barreiras, sejam elas financeiras, culturais etc. (ESCOREL ET AL, 2007). 
Nessa linha de intelecção, ilustrando o sobredito, vale observar as precisas ponderações de Starfield (2002, p. 28) acerca da atenção primária:

A atenção primária é aquele nível de um sistema de serviço de saúde que oferece a entrada no sistema para todas as novas necessidades e problemas, fornece atenção sobre a pessoa (não direcionada para a enfermidade) no decorrer do tempo, fornece atenção para todas as condições, exceto as muito incomuns ou raras, e coordena ou integra a atenção fornecida em algum outro lugar ou por terceiros. Assim, é definida como um conjunto de funções que, combinadas, são exclusivas da atenção primária. A atenção primária também compartilha características com outros níveis dos sistemas de saúde: responsabilidade pelo acesso, qualidade e custos; atenção à prevenção, bem como ao tratamento e à reabilitação; e trabalho em equipe. A atenção primária não é um conjunto de tarefas ou atividades clínicas exclusivas; virtualmente, todos os tipos de atividades clínicas (como diagnóstico, prevenção, exames e várias estratégias para o monitoramento clínico) são características de todos os níveis de atenção. Em vez disso, a atenção primária é uma abordagem que forma a base e determina o trabalho de todos os outros níveis dos sistemas de saúde. A atenção primária aborda os problemas mais comuns na comunidade, oferecendo serviços de prevenção, cura e reabilitação para maximizar a saúde e o bem-estar. Ela integra a atenção quando há mais de um problema de saúde e lida com o contexto no qual a doença existe e influencia a resposta das pessoas a seus problemas de saúde. É a atenção que organiza e racionaliza o uso de todos os recursos, tanto básicos como especializados, direcionados para a promoção, manutenção e melhora da saúde.

Portanto, a atenção primária, incluindo-se aí o PSF como componente da estrutura, lida com os problemas mais corriqueiros e menos definidos, geralmente em unidades comunitárias como centros de saúde, escolas e residências. Os pacientes/usuários têm acesso direto a uma fonte apropriada de atenção que é continuada ao longo do tempo, para diversos problemas e que compreende a necessidade de serviços preventivos.

À guisa de informação, dados do Instituto Brasileiro de Geografia e Estatística - IBGE (2008) apontam que cerca de 27,5 milhões de domicílios particulares permanentes estão cadastrados em unidade de saúde da família, num total aproximado de 57,6 milhões de domicílios brasileiros.

No tocante a infraestrutura concernente às ações da atenção básica, destaca-se a equipe multiprofissional, atuante em perspectiva interdisciplinar, com articulação das práticas e saberes de seus membros, enfrentando situações apresentadas e propondo soluções em conjunto.

A Portaria n. ${ }^{\circ}$ 648, datada de 28 de março de 2006, oriunda do Ministério da Saúde, e que "Aprova a Política Nacional de Atenção Básica, estabelecendo a revisão de diretrizes e normas para a organização da Atenção Básica para o PSF e o PACS”, aponta que a existência de uma equipe multiprofissional é necessária para a implantação das equipes de saúde da família, devendo esta ser composta por, no mínimo, médico, enfermeiro, auxiliar de enfermagem ou técnico de enfermagem e agentes comunitários de saúde.

Dentro do "universo" de profissionais que compõem a equipe de saúde da família, tem-se como ponto de grande interesse o enfermeiro, posto que constitui um dos pilares do presente trabalho.

A enfermagem, como bem descreve o Código de Ética dos Profissionais da Enfermagem (Resolução n. ${ }^{0} 311$, do Conselho Federal de Enfermagem, datada de 08 de fevereiro de 2007), “[...] é uma profissão comprometida com a saúde e a qualidade de vida da pessoa, família e coletividade". Além disso, no tocante aos profissionais da enfermagem, o aludido Código aponta:

0 profissional de enfermagem participa, como integrante da equipe de saúde, das ações que visem satisfazer as necessidades de saúde da população e da defesa dos princípios das políticas públicas de saúde e ambientais, que garantam a universalidade de acesso aos serviços de saúde, integralidade da assistência, resolutividade, preservação da autonomia das pes- 
soas, participação da comunidade, hierarquização e descentralização político-administrativa dos serviços de saúde.

O profissional de enfermagem respeita a vida, a dignidade e os direitos humanos, em todas as suas dimensões.

0 profissional de enfermagem exerce suas atividades com competência para a promoção do ser humano na sua integralidade, de acordo com os princípios da ética e da bioética.

No que se refere ao PSF, as atribuições dos seus profissionais estão definidas na já reportada Portaria n. ${ }^{0}$ $648 / 2006$, sendo que algumas delas são gerais, comuns a todos os profissionais, e outras específicas. Desse modo, são atribuições específicas do enfermeiro:

I - realizar assistência integral (promoção e proteção da saúde, prevenção de agravos, diagnóstico, tratamento, reabilitação e manutenção da saúde) aos indivíduos e famílias na USF e, quando indicado ou necessário, no domicílio e/ou nos demais espaços comunitários (escolas, associações etc), em todas as fases do desenvolvimento humano: infância, adolescência, idade adulta e terceira idade;

II - conforme protocolos ou outras normativas técnicas estabelecidas pelo gestor municipal ou do Distrito Federal, observadas as disposições legais da profissão, realizar consulta de enfermagem, solicitar exames complementares e prescrever medicações;

III - planejar, gerenciar, coordenar e avaliar as ações desenvolvidas pelos ACS;

IV - supervisionar, coordenar e realizar atividades de educação permanente dos ACS e da equipe de enfermagem;

V - contribuir e participar das atividades de Educação Permanente do Auxiliar de Enfermagem, ACD e THD; e $\mathrm{VI}$ - participar do gerenciamento dos insumos necessários para o adequado funcionamento da USF.
Destarte, as incumbências atribuídas ao enfermeiro dão conta da sua importância dentro do círculo de profissionais que integram o Programa de Saúde da Família - PSF, funcionando como uma das engrenagens indispensáveis à efetivação de toda uma sistemática, com elevado grau de autonomia, que envolve como visto uma complexidade de atividades.

Ante a perspectiva traçada, ainda com vistas às atribuições do enfermeiro, nota-se a patente necessidade de integração deste profissional com os agentes comunitários de saúde, pois são os elos entre a equipe de saúde e a população. São os agentes comunitários de saúde os responsáveis por, entre outras coisas, cadastrar os indivíduos da área de atuação, orientar as famílias quanto à utilização dos serviços de saúde disponibilizados, realizar visitas domiciliares as famílias e usuários sob sua responsabilidade etc.

Todavia, vale frisar que apesar de essencial, a integração do enfermeiro com os agentes comunitários não despreza a indispensabilidade da conexão com os outros profissionais da equipe.

Outrossim, o profissional da enfermagem atuante no âmbito do PSF deve, indispensavelmente, estar ciente do seu papel contributivo no que diz respeito a educação da comunidade a ele vinculada, além da sua ingerência na formação e capacitação de outros profissionais, mormente aqueles que compõem a equipe de enfermagem, repercutindo na melhoria da qualidade de vida dos usuários. (Borges, 2005)

Em verdade, o papel do profissional da enfermagem, no PSF foi redimensionado, ganhando novos contornos, notadamente na realização da assistência pré-natal e puerperal, conforme será abordado no tópico seguinte. 


\subsection{ENFERMEIRO E A REALIZACÃO DA ASSISTÊNCIA PRÉ-NATAL NO PROGRAMA DE SAÚDE DA FAMÍLIA - PSF}

Nos tempos hodiernos, a evolução da mulher e o seu importante papel na sociedade e no seio familiar é cada vez mais destacado. Esse quadro, por consequência, reflete nas ações de saúde referentes ao atendimento do gênero em tela.

Nesse toar, releva afirmar que as mulheres são maioria na população brasileira e as principais usuárias do Sistema Único de Saúde - SUS. Estas buscam os serviços de saúde não só para atendimento próprio, mas também na condição de acompanhantes de familiares e amigos (BRASIL, 2004, p. 9).

Em nosso país, até meados dos anos 80 , as políticas de saúde pública direcionadas à atenção da saúde da mulher eram bastante restritas, normalmente circunscritas ao ciclo gravídico-puerperal. Disso decorrem várias críticas por grupos organizados de mulheres, descontentes com a ótica restritiva das políticas públicas concernentes à saúde da mulher (Op.cit, p. 15-16).

Resulta daí a elaboração do Programa de Atenção Integral à Saúde da Mulher - PAISM, pelo Ministério da Saúde, no ano de 1983. Este programa, diga-se de passagem, anterior a Constituição Federal e, consequentemente, prévio a implantação do Sistema Único de Saúde - SUS, já contemplava uma visão de integralidade (SOUTO, 2008).

A política de saúde definida pelo PAISM orientava-se para uma assistência em diversos níveis, abrangendo as diferentes fases do ciclo vital da mulher, além de ações de planejamento familiar e ações educativas em saúde e sexualidade, exigindo da equipe multiprofissional de saúde uma nova postura de trabalho na busca da humanização da assistência (ÁVILA, 2001, p. 3).
Não se olvide que, a toda evidência, o ciclo vital da mulher tem como uma das etapas mais marcantes a gestação e o parto, demandando uma grande atenção e o devido acompanhamento ao ciclo gravídico-puerperal da mulher, sobretudo com a adequada atenção pré-natal e puerperal.

Nesse passo, ilustrando o sobredito, vale transcrever as palavras de Rios e Vieira (2007, p. 478), observe-se:

\begin{abstract}
A realização de ações educativas no decorrer de todas as etapas do ciclo grávido-puerperal é muito importante, mas é no pré-natal que a mulher deverá ser melhor orientada para que possa viver o parto de forma positiva, ter menos riscos de complicações no puerpério e mais sucesso na amamentação. Considerando o pré-natal e nascimento como momentos únicos para cada mulher e uma experiência especial no universo feminino, os profissionais de saúde devem assumir a postura de educadores que compartilham saberes, buscando devolver à mulher sua autoconfiança para viver a gestação, o parto e o puerpério.

0 período pré-natal é uma época de preparação física e psicológica para o parto e para a maternidade e, como tal, é um momento de intenso aprendizado e uma oportunidade para os profissionais da equipe de saúde desenvolverem a educação como dimensão do processo de cuidar.
\end{abstract}

Dessas ideias, exsurge lícito observar que a atenção pré-natal e puerperal visa, essencialmente, “[...] acolher a mulher desde o inicio da gravidez, assegurando, no fim da gestação, o nascimento de uma criança saudável e a garantia do bem-estar materno e neonatal” (BRASIL, 2005, p. 10).

Destaque-se, oportunamente, que o profissional da enfermagem, inserido nesse contexto, possui não somente o embasamento teórico-cientifico, mas também o amparo legal para prestar a assistência pré-natal de baixo risco (Decreto n. ${ }^{\circ}$ 94.406/87). Sendo assim, forçoso afirmar que o desenvolvimento adequado das suas competências contribui de modo significativo para a qualidade de vida materna e infantil. 
Bem por isso, os profissionais da enfermagem devem estar antenados nesse objetivo maior. Feliz, assim, a seguinte colocação:

A qualificação permanente da atenção ao pré-natal, ao parto e ao puerpério deve sempre ser perseguida na perspectiva de garantir uma boa condição de saúde tanto para a mulher quanto para o recém-nascido, bem como de possibilitar à mulher uma experiência de vida gratificante nesse período. Para isso, é necessário que os profissionais envolvidos em qualquer instância do processo assistencial estejam conscientes da importância de sua atuação e da necessidade de aliarem o conhecimento técnico específico ao compromisso com um resultado satisfatório da atenção, levando em consideração o significado desse resultado para cada mulher. A consulta pré-natal, para muitas mulheres, constitui-se na única oportunidade que possuem para verificar seu estado de saúde; assim, deve-se considerá-la também como uma chance para que o sistema possa atuar integralmente na promoção e, eventualmente, na recuperação de sua saúde (SÃO PAULO, 2010, p. 12).

A outro giro, a atenção pré-natal que, em geral, envolve procedimentos simples, deve contar com a dedicação do enfermeiro no tratamento da gestante, disposto a ouvi-la, oferecer-lhe o apoio necessário, estabelecendo uma relação de confiança, ajudando-a a conduzir a experiência da maternidade com máxima independência.

Sistematicamente, as principais atribuições do enfermeiro no processo de atenção à gestante e à puérpera no âmbito do Programa de Saúde da Família - PSF são: realização de consulta pré-natal de baixo risco; solicitação de exames de rotina e orientação de tratamento conforme protocolo de serviço; registro de atendimento no prontuário e no cartão da gestante a cada consulta; encaminhamento de gestantes classificadas como de risco para consulta com o profissional médico; promoção de atividades educativas na unidade para as mulheres e seus familiares, reuniões de grupos de sala de espera, etc.; realização de coleta de exame colpocitológico; realização de visitas domiciliares de acordo com a rotina da unidade (Op. cit., p. 20).
A partir do momento que resta confirmada a gravidez, há o primeiro contato da mulher grávida (e, idealmente, do seu acompanhante) com o enfermeiro, o que constitui o cognominado acolhimento, com a realização das orientações sobre a assistência pré-natal e, principalmente, com a averiguação das impressões da gestante no tocante a gestação.

Logo, o profissional da enfermagem deve ter em vista que a gravidez é um acontecimento complexo, envolvendo, entre tantos, aspectos psicológicos, biológicos, sociais, comportamentais, econômicos, socioculturais e que implica em profundas alterações físicas e emocionais na mulher. Por isso, também, a necessidade do acompanhamento profissional continuo e, sobretudo, humanístico.

Bem por isso, na busca por uma assistência mais humanizada, complementando-se o atendimento realizado nas consultas, tem-se adotado, não raras vezes, durante o período pré-natal, estratégias diversas (como cursos, grupos, aulas etc.), com o escopo de ajudar a gestante/parturiente e seus familiares a lidar com as vivências, com os cuidados necessários durante o ciclo gravídico-puerperal da mulher, bem como com a preparação para o parto e a maternidade (BORGES, 2005, p. 51).

Trata-se, em verdade, de ações de cunho educativo, atribuição do enfermeiro, que contribuem para que as gestantes (e, idealmente, em conjunto com familiares) conheçam a importância do pré-natal na redução da morbi-mortalidade materno-fetal, cientifiquem-se das rotinas necessárias para um pré-natal de qualidade e das transformações físicas e psíquicas. Além disso, tais ações devem estimular o aleitamento materno, orienta sobre exercícios posturais, advertir sobre as consequências e danos decorrentes do uso abusivo de drogas ou da automedicação, desconstituir medos e fantasias referentes à gestação e ao parto, etc.

Abordando o contato inicial com a gestante, não é demais lembrar, será este o momento para forne- 
cimento do o cartão da gestante (devidamente identificado e preenchido), ocasião para concretização das recomendações a respeito de vacinas, atividades físicas, atividades educativas, solicitações de exames complementares, etc.

Por seu turno, deve ser agendada a primeira consulta pré-natal, em tempo suficiente para que possam ser realizados os exames solicitados e recebidos os seus resultados, estabelecendo-se, conforme cada caso, os próximos passos. É preciso, pois, situar que nessa primeira consulta identificam-se os eventuais riscos na gravidez (seja para a própria gestante ou para o feto), o que deverá ser reavaliado a cada retorno.

Nesse diapasão, vale dizer que os fatores de risco na gravidez estão insertos em grupos de características que devem ser cuidadosamente sopesados, como, por exemplo, características individuais e condições sócio demográficas desfavoráveis (idade menor que 15 e maior que 35 anos, situação familiar insegura e não aceitação da gravidez, situação conjugal insegura, dependência de drogas lícitas ou ilícitas, etc.), histórico reprodutivo anterior (abortamento habitual, síndromes hemorrágicas, cirurgia uterina anterior, pré-eclâmpsia/ eclampsia, etc.), doença obstétrica na gravidez atual (ganho ponderal inadequado, isoimunização, hemorragias na gestação, etc.) e intercorrências clínicas (cardiopatias, nefropatias, hemopatias, epilepsia, pneumopatias, etc.) (BRASIL, 2005, p. 22-24).

Tendo em vista que o profissional da enfermagem possui o embasamento teórico-cientifico e o amparo legal para prestar somente a assistência pré-natal de baixo risco, identificando-se um ou mais dos supramencionados fatores, deve ser realizado o pertinente encaminhamento da gestante, seja para tratamento no âmbito do próprio PSF ou para tratamento na atenção especializada que, após avaliação, procedera com o retorno da gestante para a atenção básica com as recomendações para seguimento da gravidez ou, em caso diverso, manterá a assistência pré-natal nos serviços de referencia para gestação de alto risco. (Op. cit., p. 24).

Recomenda-se, geralmente, o número mínimo de seis consultas, sendo, preferencialmente, uma no primeiro trimestre, duas no segundo trimestre e três no último trimestre, todas realizadas na esfera da unidade de saúde ou durante visitas domiciliares. Desse modo, "a maior frequência de visitas no final da gestação visa à avaliação do risco perinatal e das intercorrências clínico-obstétricas mais comuns nesse trimestre, como trabalho de parto prematuro, pré-eclâmpsia e eclâmpsia, amniorrexe prematura e óbito fetal” (SÃO PAULO, 2010, p.56).

Some-se a isso que além das consultas abordadas, deve haver ao menos um retorno puerperal. Ademais, em todas essas consultas, sejam elas pré-natais ou retornos puerperais, a qualidade na sua realização será ponto chave, identificando eventuais problemas passíveis de causar algum risco a mulher ou a criança.

Enfim, a realização de pré-natal de qualidade influi de forma patente na redução das taxas de mortalidade materna, ou seja, assegura a ocorrência de um parto sem intercorrências, obstando fortuitos prejuízos à saúde da mãe e do recém-nascido. Assim, sendo, o profissional da enfermagem deve oferecer todo o suporte devido, assegurando a consecução dos resultados desejados. 


\section{CONCLUSÃO}

Em linhas conclusivas, observou-se, a partir do conteúdo do presente trabalho, a evolução das políticas públicas concernentes à saúde dos indivíduos, mormente com a elaboração do PSF, após a implantação do SUS, fruto da Constituição Federal.

A equipe multidisciplinar que compõe 0 atendimento prestado no âmbito do PSF tem no enfermeiro um dos seus grandes pilares, comprometido com a saúde e qualidade de vida da população (pessoa, família e coletividade). Este profissional, ainda com vistas ao PSF, teve sua participação acentuada, ganhando uma maior autonomia na realização das atividades.

Nessa nova perspectiva, destacam-se os cuidados com a saúde da mulher, especialmente com a implantação de novas ações como o Programa de Atenção Integral à Saúde da Mulher (PAISM), contemplando a assistência nas diferentes fases do seu ciclo vital, demandando uma nova postura por parte do enfermeiro.

Resulta daí o despertar para o valor do enfermeiro, com o devido preparo técnico-cientifico e o pertinente respaldo legal para o desenvolvimento da assistência pré-natal de baixo risco, contribuindo de modo expressivo na qualidade de vida da mãe e do recém-nascido.

Fundam-se, as atribuições do enfermeiro, nessa toada, em um processo de inestimável importância, compreendendo a realização de consulta pré-natal, solicitação de exames de rotina, promoções de ações educativas e outras mais. Tais atividades, embora se reportem a procedimentos aparentemente simplistas, constituem o núcleo das atribuições do enfermeiro na consulta pré-natal realizada no âmbito do PSF.

Logo, os profissionais da enfermagem devem, indispensavelmente, ter consciência do seu papel no PSF, debruçando-se de forma satisfatória sobre as atividades de acompanhamento do ciclo gravídico-puerperal da mulher, conscientes das variadas transformações resultantes da gravidez, avaliando não somente as questões diretamente ligadas à saúde da mulher, mas também questões laterais, como a situação familiar, aceitação e compreensão da gravidez, situação conjugal, etc.

Portanto, o superdimensionamento do papel do enfermeiro na realização do pré-natal no PSF, que lhe trouxe maior reconhecimento, constitui-se, também, em uma grande responsabilidade, não podendo o profissional distanciar-se das suas importantes incumbências, visando sempre a qualidade de vida da mãe e recém-nascido.

Eis o que esperam as mulheres, usuárias dos serviços de saúde prestados no âmbito do Programa de Saúde da Família, que carregam consigo a inenarrável experiência vivenciada durante o ciclo gravídico-puerperal, contando com a assistência continuada de profissionais da enfermagem comprometidos com a sadia qualidade de vida da mãe e do recém-nascido. 


\section{REFERÊNCIAS}

ÁVILA, Maria Betânia (coord.). Avaliação da implementação do Programa de Assistência Integral à Saúde da Mulher (PAISM) nos Municípios: estudo de caso no Município de Recife. SOS Corpo - Instituto Feminino para a Democracia, Recife, PE. 2001. Disponível em: <http://www.soscorpo.org.br/Adm/userfiles/PAISM\%20em\%20 Recife.pdf>. Acesso em: 12. jun. 2011.

BORGES, Cristiane José. Avaliação de ações educativas em saúde com grupos de gestantes: estudo comparativo entre unidade saúde da família e unidade básica de saúde. 2005. Dissertação (Mestrado em Enfermagem). Universidade Federal de Goiás, Goiânia, 2005. Disponível em: <http://www.bdtd.ufg.br/tedesimplificado/tde_ busca/arquivo.php?codArquivo=31>. Acesso em: 04 jul. 2011.

BRASIL. Lei n. ${ }^{0} 2.604$, de 17 de setembro de 1955. Regula o exercício da enfermagem profissional. Diário Oficial [da] República Federativa do Brasil, Brasília, DF. 21 set. 1955. Disponível em: <http://www.planalto.gov. br/ccivil_03/Leis/L2604.htm>. Acesso em: 26 jun. 2011.

BRASIL. Ministério da Saúde. Secretaria de Atenção à Saúde. Departamento de Ações Programáticas Estratégicas. Política nacional de atenção integral à saúde da mulher: princípios e diretrizes. Brasília: Ministério da Saúde, 2004.

BRASIL. Ministério da Saúde. Secretaria de Atenção à Saúde. Departamento de Ações Programáticas Estratégicas. Área Técnica de Saúde da Mulher. Pré-natal e puerpério: atenção qualificada e humanizada. Brasília: Ministério da Saúde, 2005.

BRASIL. Ministério da Saúde. Gabinete do Ministro. Portaria n. ${ }^{0}$ 648, de 28 de março de 2006. Aprova a Política Nacional de Atenção Básica, estabelecendo a revisão de diretrizes e normas para a organização da Atenção Básica para o Programa Saúde da Família (PSF) e o Programa Agentes Comunitários de Saúde (PACS). Diário Oficial [da] República Federativa do Brasil, Brasília, DF. 29 mar. 2006. Disponível em: <http://bvsms.saude. gov.br/bvs/saudelegis/gm/2006/prt0648_28_03_2006.html>. Acesso em: 26 jun. 2011.

CONSELHO Federal de Enfermagem. Resolução n. ${ }^{\circ}$ 311, de 08 de fevereiro de 2007. Código de Ética Profissional de Enfermagem. Disponível em: <http://www.portalcofen.gov.br/sitenovo/node/4158>. Acesso em: 17 jun. 2011.

ESCOREL, Sarah et al . O Programa de Saúde da Família e a construção de um novo modelo para a atenção básica no Brasil. Rev Panam Salud Publica, Washington, v. 21, n. 2-3, Mar. 2007 . Disponível em: <http://www. scielosp.org/scielo.php?script=sci_arttext\&pid=S1020-49892007000200011\&lng=en\&nrm=iso>. Acesso em: 5 jul. 2011.

INSTITUTO Brasileiro de Geografia e Estatística. Um panorama da saúde no Brasil: acesso e utilização dos serviços, condições de saúde e fatores de risco e proteção à saúde. 2008. Disponível em: <http://www.ibge.gov. br/home/estatistica/populacao/panorama_saude_brasil_2003_2008/default.shtm>. Acesso em: 3 jul. 2011. 
RIOS, Claudia Teresa Frias; VIEIRA, Neiva Francenely Cunha. Ações educativas no pré-natal: reflexão sobre a consulta de enfermagem como um espaço para educação em saúde. Ciência e saúde coletiva, Rio de Janeiro, RJ, v. 12, n. 2, p. 477-486, mar./abr. 2007. Disponível em: <http://redalyc.uaemex.mx/pdf/630/63012221.pdf>. Acesso em: 15 jul. 2011.

SÃO PAULO (Estado). Secretaria da Saúde. Coordenadoria de Planejamento em Saúde. Assessoria Técnica em Saúde da Mulher. Atenção à gestante e à puérpera no SUS-SP: manual técnico do pré-natal e puerpério. São Paulo: SES/SP, 2010.

SEVERINO, Antônio Joaquim. Metodologia do trabalho científico. 23. ed. rev. atual. São Paulo: Cortez, 2007.

SOUTO, Kátia Maria Berreto. A política de atenção integral à saúde da mulher: uma análise de integralidade e gênero. Revista Ser Social, Brasília, DF, v. 10, n. 22, jan./jun. 2008. Disponível em: <http://www.red.unb.br/ index.php/SER_Social/article/view/17/18 >. Acesso em: 3 jun. 2011.

STARFIELD, Barbara. Atenção primária: equilíbrio entre necessidades de saúde, serviços e tecnologia. Brasília: UNESCO, Ministério da Saúde, 2002.

VIANA, Ana Luiza D’ávila; DAL POZ, Mario Roberto. A reforma do sistema de saúde no Brasil e o Programa de Saúde da Família. Physis, Rio de Janeiro, v. 8, n. 2, Dec. 1998. Disponível em: <http://www.scielo.br/scielo. php?script=sci_arttext\&pid=S0103-73311998000200002\&lng=en\&nrm=iso>. Acesso em: 13 jul. 2011.

Recebido em: 22 de Abril de 2013 Avaliado em: 1 de Agosto de 2013 Aceito em: 29 de Julho de 2013
1. Enfermeira e especialista em Enfermagem ginecológica e obstetrícia pela Universidade Tiradentes - UNIT, Aracaju, SE, Brasil. E-mail: brigida-cabral@hotmail.com

2. Doutoranda do Programa de Pós-Graduação do Departamento de Ginecologia e Obstetrícia da Faculdade de Medicina de Ribeirão da Universidade de São Paulo (FMRP-USP). Mestre em Ginecologia e Obstetrícia da Faculdade de Medicina de Ribeirão da Universidade de São Paulo (FMRP-USP). Especialista em Saúde da Mulher pela Universidade de Ribeirão Preto (UNAERP) e Fisioterapeuta pela Universidade Tiradentes (UNIT). Ribeirão Preto, SP, Brasil.

3. Doutorado em andamento pelo departamento de Ginecologia e Obstetrícia da Faculdade de Medicina de Ribeirão Preto da Universidade de São Paulo - FMRP/USP. Mestrado em Ginecologia e Obstetrícia pela Universidade de São Paulo . Professor da Universidade Tiradentes, Brasil 